\title{
Assessment of SGS closure for isochoric combustion of hydrogen-air mixture
}

\author{
G. Ghiasi ${ }^{a}$,* N. A. K. Doan ${ }^{\mathrm{a}}$, N. Swaminathan ${ }^{\mathrm{a}}$, B. Yenerdag ${ }^{\mathrm{b}}$, Y. \\ Minamoto $^{\mathrm{b}}$, M. Tanahashi ${ }^{\mathrm{b}}$ \\ ${ }^{a}$ Department of Engineering, Cambridge University, Cambridge CB2 1PZ, UK \\ ${ }^{b}$ Department of Mechanical Engineering, Tokyo Institute of Technology, 2-12-1 \\ Ookayama, Meguro-ku, Tokyo 152-8550, Japan \\ (Submitted to J. Hydrogen Energy February 15, 2018)
}

\begin{abstract}
Direct numerical simulation (DNS) data of freely propagating turbulent premixed flame of stoichiometric hydrogen air mixture inside a closed vessel is analysed to study a sub-grid combustion closure based on unstrained flamelet approach. This modelling framework needs closures for the sub-grid scale (SGS) reaction rate and scalar dissipation rate. The results show that the closure models for these two SGS quantities work quite well. The dissipation rate closure involves a scale dependent parameter, $\beta_{c}$, which is related to the flame curvature induced effects. The reactivity of reactant mixture increases with time in isochoric combustion because the mixture temperature and pressure increases with time. This also influences the parameter $\beta_{c}$ and thus the dynamic evaluation of this parameter is investigated using the DNS data.
\end{abstract}

Keywords: DNS, isochoric combustion, Flamelets, SDR, SGS combustion

*gg381@cam.ac.uk

Preprint submitted to International Journal of Hydrogen Energy $\quad$ February 15, 2018 


\section{Introduction}

The performance and characteristics of Internal Combustion (IC) engines can be improved using various concepts such as Homogeneous Charge Compression Ignition (HCCI), Port fuel injection (PFI), and Direct Injection

${ }_{5}$ (DI). These have the potential to reduce in-cylinder pollutant formation and increase the overall efficiency. However, achieving these goals is challenging and an improved understanding of the underlying physics of turbulent combustion in IC engines operating with the above concepts is necessary. Hydrogen is recognised as an attractive and a promising fuel for future main stream IC engines and gas turbines. If it is used with optimised engine operating conditions, there are emission benefits, including elimination of soot, unburned hydrocarbons, and greenhouse gases. Aside from pollutant reductions, hydrogen is also recognised as an important fuel because it can be derived from various renewable sources. Thus, understanding turbulent combustion of hydrogen and its modeling, in general, is important.

The advent of computational hardware, methodologies and modeling approaches in recent times make the computational fluid dynamics (CFD) as an important and cost-effective tool to gather required insights on the incylinder flow, combustion and their interactions. Direct Numerical Simulation (DNS) resolves all the relevant length and time scales involved in turbulent reacting flows without any modeling assumptions. Thus, DNS can be considered as numerical experiments helping us to gather fundamental insights and thereby provide unique opportunities to test and validate modeling hypotheses for turbulence-chemistry interactions. This is because all 
from DNS data. However, the computational burden of including complex engine geometries and flow paths preclude the DNS for engine flows. Many DNS studies have been conducted in the past to investigate hydrogen-air turbulent premixed flames in a variety of flame and flow configurations, such as planar [1-4] and oblique("Vee") [5, 6] flames propagating in homogeneous turbulence, swirling flames [7-9] and jet flames [10, 11]. Many useful insights are deduced from these studies for isobaric combustion. In IC engines, combustion occurs at constant volume under pressure rising conditions and thus, the reactant temperature and pressure change with time over wide ranges, 35 which can be mimicked by simulating turbulent combustion inside a vessel of fixed volume.

Yenerdag et al. [12, 13] conducted such a simulation and considered hydrogen-air premixed flame initiated using an initial spherical kernel and propagating in a turbulent flow to mimic the combustion processes observed in an IC engine with its piston at the top dead centre (TDC). However, the pressure rise during the combustion in the DNS was not as big as in an IC engine but sufficient to address the objectives of this investigation, which are elaborated later in this section.

Unsteady Reynolds averaged Navier-Stokes (URANS) simulations are used extensively to simulate in-cylinder turbulent reacting flows in industries because of its computational cost. Large Eddy Simulations (LES) are, however, becoming feasible for combusting flows inside IC engines with intricate geometry because of the recent advances in computational capabilities. This helps to investigate cycle-to-cycle variations. The validity of these simu50 lations depends on the closures used to describe turbulence, combustion and 
their interactions. The combustion modeling, its accuracy and robustness play a vital role in this.

The currently emerging Large Eddy Simulation (LES) models for engine flow exhibit large uncertainties with respect to the subgrid scale (SGS) models [14] and combustion is a SGS phenomenon. Various combustion closures developed in the past to tackle turbulent premixed combustion are reviewed and discussed in [15 17]. Out of these many modeling methods, flamelet based methods are quite attractive for engine application as they are relatively simple and computationally less expensive. Flame surface density based models have also been used in past LES studies of premixed flames in simple enclosures [18 21], and also in engines (see [14] for a review). The progress and application of LES for engine flows has been reviewed in [14, 22 24] noting that (i) LES requires a low level of empiricism, (ii) advanced subgrid turbulence model can ease computational grid requirement [14] but will increase computational cost and (iii) the SGS combustion models will have adequate fidelity if their parameters were closely tied to the physics of the problem [23, 24]. One such combustion closure, called as FlaRe (Flamelets revised for physical consistencies) is developed at Cambridge University using flamelets and this method is of interest for this study.

The FlaRe approach discussed briefly in section 2 is based on unstrained flamelets and is investigated here using DNS data of turbulent combustion of hydrogen-air mixture in pressure rising condition. This model is based on tabulated chemistry approach and maintains physical consistencies among various SGS processes in turbulent combustion (see section 2) since the 75 model parameters are linked directly to these (flow and thermo-chemical) 
processes. Thus, these parameters can be estimated based on the local turbulence and thermo-chemical information available within the simulation paradigm (URANS or LES). This model has been tested for laboratory scale flames [25-29] and practical burners [30, 31] including engine relevant flow and flame conditions [32]. Its capability for IC engine simulation has also been tested in [33, 34] using URANS approach.

Its subgrid scale version has been developed and tested for premixed [35, 36] and partially premixed [37] flames. This approach has a scale dependent parameter, $\beta_{c}$, to include effects arising from flame curvature related phe85 nomena, which are strongly related to the SGS thermo-chemical processes. Since the in-cylinder reactant temperature and pressure vary with time, the mutual influence of flame curvature and thermo-chemical activities may be stronger compared to those in isobaric combustion and thus the $\beta_{c}$ value is likely to change with time.

A dynamic procedure for $\beta_{c}$ was developed and tested using DNS data [38, 39] and its behaviour in LES was also assessed by comparing LES statistics [39] with measurements in turbulent Bunsen flames. Langella et al. [39] found small difference in the Bunsen flames statistics obtained using an empirically determined (static) $\beta_{c}$ value and the dynamic procedure. This could be because (i) the empirical value was chosen carefully and (ii) combustion in the Bunsen flames occurs at constant pressure with no changes in the temperature and pressure of the incoming reactant. As noted earlier, the thermodynamic conditions of the reactant mixture change with time in isochoric combustion because of the compression caused by the expanding gases. ${ }_{100}$ This is similar to that occurring inside an IC engine. Thus, the objectives of 
this study are (i) to test the unstrained flamelet closure for the SGS reaction rate and (ii) to investigate the behaviour of $\beta_{c}$ and its dynamic evaluation for constant volume turbulent combustion of hydrogen-air mixture. These objectives are achieved by analysing the DNS data of isochoric combustion [12, 13].

This paper is organised as follows. The SGS combustion modeling is discussed briefly in section 2. The detail of DNS data and its processing are explained in section [3. The results are discussed in section 4 and the conclusions are summarised in the final section.

\section{SGS Combustion Modeling Investigated}

A reaction progress variable is typically used to track the progress of premixed combustion and this variable is defined using either temperature or scalar mass fractions. Here, it is defined using water mass fraction, $Y_{\mathrm{H}_{2} \mathrm{O}}$,

$$
c=\frac{Y_{\mathrm{H}_{2} \mathrm{O}}-Y_{\mathrm{H}_{2} \mathrm{O}}^{u}}{Y_{\mathrm{H}_{2} \mathrm{O}}^{b}-Y_{\mathrm{H}_{2} \mathrm{O}}^{u}},
$$

110 tively. Since $Y_{\mathrm{H}_{2} \mathrm{O}}^{u}=0$ for the cases investigated here, the above definition simplifies to $c=Y_{\mathrm{H}_{2} \mathrm{O}} / Y_{\mathrm{H}_{2} \mathrm{O}}^{b}$. Thus, $c$ varies from 0 in the reactant mixture to 1 in the products. The transport equation for its Favre-filtered value, $\widetilde{c}$, is

$$
\bar{\rho} \frac{D \widetilde{c}}{D t}=\frac{\partial}{\partial x_{i}}\left(\overline{\rho \mathcal{D} \frac{\partial c}{\partial x_{i}}}\right)-\frac{\partial}{\partial x_{i}}\left(\bar{\rho} \widetilde{u_{i} c}-\bar{\rho} \widetilde{u_{i}} \widetilde{c}\right)+\overline{\dot{\omega}_{c}}
$$

using standard notations with $\mathcal{D}$ as the molecular diffusivity of $c$ and $D / D t$ 115 is the substantial derivative. The filtered reaction rate, $\overline{\dot{\omega}_{c}}$, is modelled using

$$
\overline{\dot{\omega}_{c}}=\bar{\rho} \int_{0}^{1} \frac{\dot{\omega}_{c}(\zeta)}{\rho} \widetilde{p}\left(\zeta ; \widetilde{c}, \sigma_{c, s g s}^{2}\right) d \zeta
$$


where $\zeta$ is the sample-space variable for $c$. The SGS Favre probability density function (pdf) of $c$ is denoted as $\widetilde{p}(\zeta)$ and is modelled using a Beta function for given values of $\widetilde{c}$ and SGS variance, $\sigma_{c, s g s}^{2}$. The flamelet reaction rate, $\dot{\omega}_{c}(\zeta)=\dot{\omega}_{\mathrm{H}_{2} \mathrm{O}} / Y_{\mathrm{H}_{2} \mathrm{O}}^{b}$, is obtained by computing an unstrained laminar flame

with reactant temperature of $T_{u}$ and pressure of $P_{u}$. Also, this laminar flame has the same equivalence ratio as for the turbulent flame. The reaction rate of $\mathrm{H}_{2} \mathrm{O}, \dot{\omega}_{\mathrm{H}_{2} \mathrm{O}}$, in the laminar flame is obtained using PREMIX code of CHEMKIN-pro software. Thus, the combustion kinetics can be represented using a complex chemical mechanism. The same mechanism employed in the DNS, see section 3, is used for the laminar flame calculations of this study. The SGS variance, $\sigma_{c, s g s}^{2}=\widetilde{c}^{2}-\widetilde{c}^{2}$, is obtained in LES through its modeled transport equation, which is

$$
\bar{\rho} \frac{D \sigma_{c, s g s}^{2}}{D t} \approx \frac{\partial}{\partial x_{i}}\left[\left(\widetilde{\mathcal{D}}+\mathcal{D}_{T}\right) \frac{\partial \sigma_{c, s g s}^{2}}{\partial x_{i}}\right]-2 \bar{\rho} \widetilde{\epsilon_{c}}+2\left(\overline{\dot{\omega}_{c} c}-\overline{\dot{\omega}_{c}} \widetilde{c}\right)+2 \mathcal{D}_{T}\left|\frac{\partial \widetilde{c}}{\partial x_{i}}\right|^{2}
$$

where $\mathcal{D}_{T}$ is the SGS eddy diffusivity. In this equation, $\overline{\dot{\omega}_{c} c}$ and $\widetilde{\epsilon_{c}}$ require closures and the former is closed using a model similar to that in Eq. (3) as noted in [36, 39]. The SGS scalar dissipation rate (SDR), $\widetilde{\epsilon}_{c}$, is modelled using an algebraic expression investigated in earlier studies [38 40]:

$$
\widetilde{\epsilon_{c}}=\mathcal{F}\left[2 K_{c} \frac{s_{L}}{\delta_{t h}}+\left(C_{3}-\tau C_{4} \mathrm{Da}_{\Delta}\right)\left(\frac{2}{3} \frac{u_{\Delta}^{\prime}}{\Delta}\right)\right] \frac{\sigma_{c, s g s}^{2}}{\beta_{c}},
$$

where $\mathcal{F}=\left[1-\exp \left(-0.75 \Delta^{+}\right)\right]$with $\Delta^{+}$as the LES filter width, $\Delta$, normalised by the laminar flame thermal thickness, $\delta_{t h}$, and $u_{\Delta}^{\prime}$ is a SGS velocity scale. The laminar flame speed is $s_{L}$ and the thermochemical parameter is $K_{c}=0.79 \tau$, where $\tau$ is the temperature rise across the flame normalised 
by $T_{u}$. The other parameters are defined as $C_{3}=1.5 \sqrt{\mathrm{Ka}_{\Delta}} /\left(1+\sqrt{\mathrm{Ka}_{\Delta}}\right)$, $C_{4}=1.1 /\left(1+\mathrm{Ka}_{\Delta}\right)^{0.4}$, where $\mathrm{Ka}_{\Delta}=\left(u_{\Delta}^{\prime} / s_{L}\right)^{3 / 2}\left(\delta_{t h} / \Delta\right)^{1 / 2}$, and $\mathrm{Da}_{\Delta}=$ $\Delta s_{L} /\left(u_{\Delta}^{\prime} \delta_{t h}\right)$. It has been established in past studies that the above parameters and their values for Eq. (5) are closely connected to certain physical

140

$$
\bar{\rho} \widetilde{N_{c}}=\bar{\rho} \widetilde{\mathcal{D}}(\nabla \widetilde{c} \cdot \nabla \widetilde{c})+\bar{\rho} \widetilde{\epsilon_{c}}=\psi_{1}+\frac{\psi_{2}}{\beta_{c}} .
$$

where $\psi_{2}$ is defined by equating $\psi_{2} / \beta_{c}$ to Eq. (5). By applying a test filter to the above equation one gets

$$
\begin{gathered}
\widehat{\bar{\rho} \widehat{N}_{c}}=\widehat{\psi_{1}}+\widehat{\psi_{2} / \beta_{c}}, \\
\text { and } \widehat{\bar{\rho}} \widehat{\widehat{N_{c}}}=\widehat{\bar{\rho}} \widehat{\widetilde{\mathcal{D}}}(\nabla \widehat{\widetilde{c}} \cdot \nabla \widehat{\widetilde{c}})+\widehat{\psi_{2}} / \beta_{c} .
\end{gathered}
$$

The notation $\widehat{\widehat{\psi_{2}}}$ implies that $\psi_{2}$ must be evaluated using the test filtered quantities. The scale similarity concept suggests that $\widehat{\bar{\rho} \widehat{N}_{c}} \approx \widehat{\bar{\rho}} \widehat{\widetilde{N}}_{c}$ and hence, 
by using Eqs. (77) and (마) one gets

$$
\beta_{c}=\frac{\widehat{\widehat{\psi_{2}}}}{\widehat{\psi_{1}}+\widehat{\psi_{2} / \beta_{c}}-\widehat{\bar{\rho}} \widehat{\overrightarrow{\mathcal{D}}}(\nabla \widehat{\widetilde{c}} \cdot \nabla \widehat{\widetilde{c}})} .
$$

Equation (9) can be solved iteratively to obtain $\beta_{c}$ if other quantities in this equation are known and these are readily available in LES. For this study, they are extracted from the DNS data described next.

\section{DNS data and processing}

Yenerdag et al. [12, 13] directly simulated isochoric turbulent combustion of stoichiometric hydrogen-air mixture inside a vessel. The size of this vessel (computational domain) is $L_{x} \times L_{y} \times L_{z}=20 \times 5 \times 20 \mathrm{~mm}^{3}$ and is discretised using $N_{x} \times N_{y} \times N_{z}=1025 \times 257 \times 1025$ grid points ensuring that there are enough points in the Kolmogorov lengthscale, $\eta$, and the flame thickness. This rectangular computational domain is a simple idealized model of an IC engine combustion chamber when the piston is at the TDC. Although this combustion volume is smaller than that in a practical engine, the present configuration is chosen to keep the DNS computationally affordable allowing one to investigate the effects of time-varying reactant mixture temperature 
and pressure on flame propagation and its interaction with turbulence. The vessel's walls are specified to have a temperature of $T_{w}=450 \mathrm{~K}$ and the initial pressure in the vessel is set to be $0.1 \mathrm{MPa}$.

The flame is initiated using a spherical ignition kernel placed at the centre of the computational domain and has an initial temperature, $T_{\mathrm{ini}}$, distribution given by

$$
T_{\mathrm{ini}}=T_{u}+\left(T_{p}-T_{u}\right) \exp \left(-\frac{r^{2}}{2 R^{2}}\right),
$$
mechanism with the specific reactions involved are available in [45]. Further 
detail on the numerical simulation such as conservation equations, numerical scheme, etc., can be found in [12, 13].
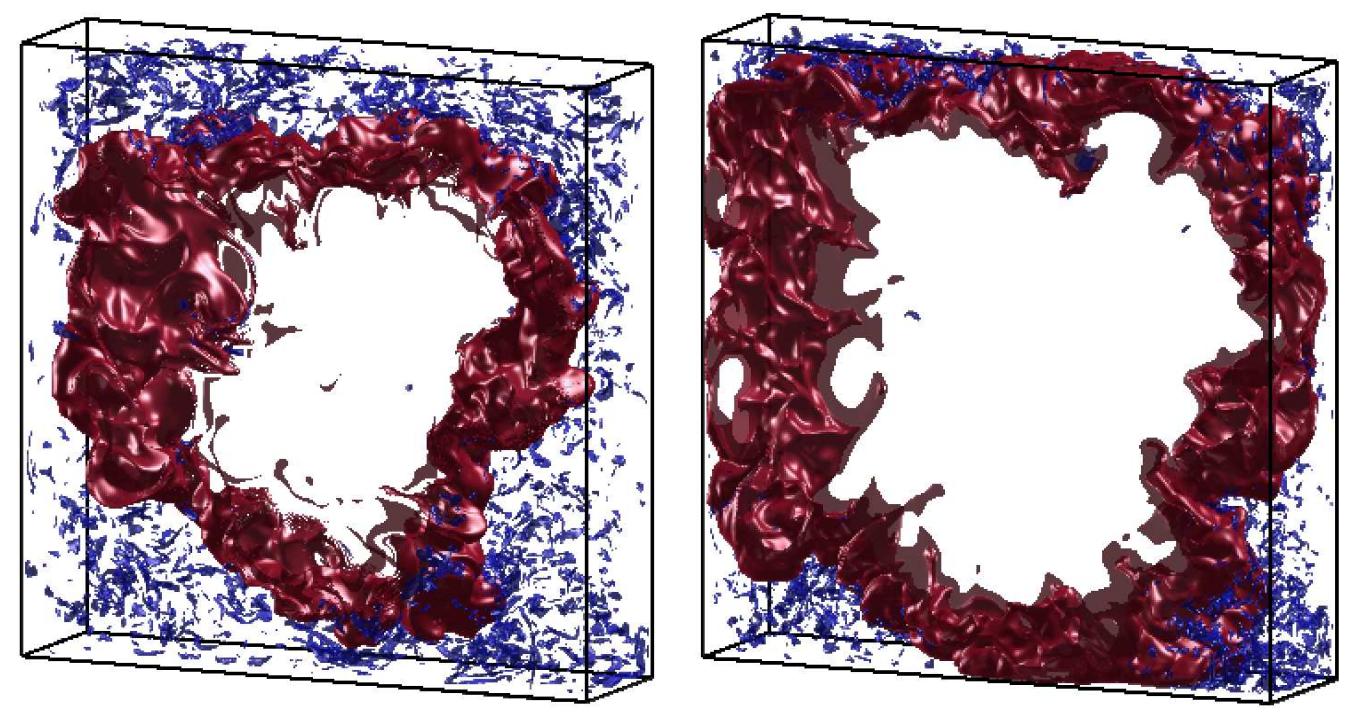

Figure 1: Visualisation of flame and flow features at $\widehat{t}=0.73$ (left) and 0.91 (right), where $\widehat{t}$ is time normalised using the turbulence integral eddy turnover time. Blue isosurfaces represent $Q=\langle Q\rangle+3 \sigma_{Q}$ and red iso-surface is for $60 \%$ of the maximum $\dot{\omega}_{c}$.

Typical flame surface and turbulence characteristics extracted at two different times, normalised using the initial eddy turnover time $t_{\text {ed }}=\ell / u^{\prime}$, are shown in Fig. 1. The flame surface is marked using a reaction rate, $\dot{\omega}_{c}$, of $60 \%$ of the maximum value at a given instant. The turbulence structure is visualised using $Q=\left(\Omega_{i j} \Omega_{i j}-S_{i j} S_{i j}\right) / 2$, where $\Omega_{i j}$ and $S_{i j}$ are the anti-symmetric and symmetric part of the strain tensor $\partial u_{i} / \partial x_{j}$. The fluid velocity in the direction $i$ is denoted using $u_{i}$. The iso-surfaces of $Q$ shown in the figure are for $\langle Q\rangle+3 \sigma_{Q}$, where $\langle Q\rangle$ is the volume averaged value of $Q$ and $\sigma_{Q}$ is its standard deviation. The turbulent structures are seen typically 
outside the flame because of the effects of heat release rate and pressure variation across the flame front. Also, these structures are not seen in the burnt mixture because of increase in the viscosity due to high temperature. The flame surface wrinkling are produced by the fine scale turbulence structures as noted in [12]. The flame propagation with time is obvious in Fig. 11 and it has not interacted with the walls yet.
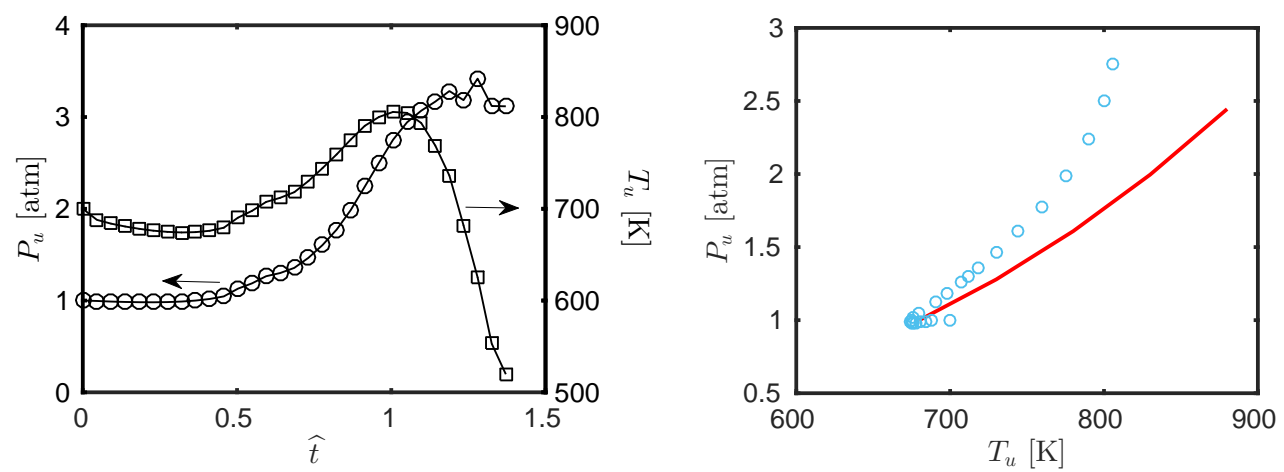

Figure 2: Temporal variation of mean pressure (o) and reactant temperature ( $\square$ ) inside the vessel is shown on the left. The picture on the right compares the $P_{u}-T_{u}$ map from the DNS (symbols) to that of isentropic theoretical curve (line).

Figure 2 shows the temporal variation of mean pressure and reactant temperature inside the vessel. The DNS is conducted for about 1.5 eddy turnover time by which time the entire charge in the vessel is consumed by the turbulent combustion. The 1.5 eddy turnover time corresponds to about 24 laminar flame time and thus the turbulence-chemistry interaction has evolved sufficiently for model testing. The pressure, $P_{u}$, shown in the figure is averaged over the entire volume but the temperature is conditionally averaged for $c \leq 0.05$ to include only the unburnt mixture. The reactant temperature decreases initially by about 30 to $40 \mathrm{~K}$ and increases after $\widehat{t} \simeq 0.4$. The 
mean pressure also shows a similar tendency as one would expect for isochoric combustion. This is because the heat release rate is relatively smaller than the heat loss through the walls in the initial stages. The increase in $P_{u}$ is because of the compression caused by the expanding hot gases which is ideally an isentropic process, but the heat loss through the walls makes it to be non-isentropic. A comparison of $P_{u}-T_{u}$ map with the theoretical curve 225 is also shown in Fig. 2. The approximation of isentropic compression is quite good at the very early stages. The irreversible chemical changes and viscous heating causes $\partial P_{u} / \partial T_{u}$ to be larger for the DNS compared to the theoretical value. The irreversibleties increase with temperature which leads to progressively, in time, increased deviation from the idealised behaviour. 230 Also, the increase in $T_{u}$ for a given increase in pressure is smaller for the DNS because of heat loss through the walls. This heat flux predominantly coming from the burnt mixture was shown to increase with $\widehat{t}$ in the earlier study [13] and thus the $P_{u}-T_{u}$ curve from the DNS deviates progressively, with deviations increasing with time, from the theoretical curve as seen in 235 Fig. 2,

To summarise, the DNS data captures the thermo-chemical processes and their interaction with turbulence which are expected inside an IC engine although the pressure rise is not as high as in a practical engine. This is because of smaller total energy released in the DNS compared to that in an engine. Since the fundamental processes are captured in the DNS, one can use it to investigate SGS combustion process and its interaction with turbulence. Hence, this data is post-processed using the steps described next to extract SGS information relevant for the modeling discussed in the previous section. 


\subsection{Data processing}

245 the DNS data is filtered explicitly using a Gaussian filter to extract various quantities of interest and density weighted filtering is used here. This filtering is performed within a region of size $2 \Delta \times 2 \Delta \times 2 \Delta$ centred at a filtering point, x. A filtered quantity of interest, $F$, is then computed as

$$
\widetilde{F}(\mathbf{x}, t)=\frac{1}{\bar{\rho}(\mathbf{x}, t)} \int_{\mathbf{x}-\Delta}^{\mathbf{x}+\Delta} \rho\left(\mathbf{x}^{\prime}, t\right) F\left(\mathbf{x}^{\prime}, t\right) \mathcal{G}\left(\mathbf{x}-\mathbf{x}^{\prime} ; \Delta\right) d \mathbf{x}^{\prime}
$$
where $\rho$ and $\bar{\rho}$ are respectively the unfiltered and filtered density and $\mathbf{x}^{\prime}$ is the sampling point in the filter sub-space. The Gaussian filter kernel, $\mathcal{G}$, is [46]

$$
\mathcal{G}\left(\mathbf{x}-\mathbf{x}^{\prime}\right)=\left(\frac{6}{\pi \Delta^{2}}\right)^{3 / 2} \exp \left(\frac{-6\left(\mathbf{x}-\mathbf{x}^{\prime}\right)^{2}}{\Delta^{2}}\right) .
$$

The SGS Favre variance of $F$ is obtained using

$$
\sigma_{F, s g s}^{2}(\mathbf{x}, t)=\widetilde{F}^{2}-\widetilde{F}^{2}
$$

The test filtered quantities required for Eqs. (9) and (10) are obtained following the above procedure but with a filter of size $\widehat{\Delta}=2 \Delta$.

\section{Results and Discussion}

Figure 3 shows typical reaction rate, $\dot{\omega}_{c}$, contours extracted from the DNS data at $\widehat{t}=0.73$ for three different values of the filter width, $\Delta^{+}=$ $\Delta / \delta_{t h}=0.5,1$ and 2. The reaction rates are normalised using $\rho_{u}, s_{L}$ and $\delta_{t h}$ for the reactant mixture with $T_{u}$ and $P_{u}$ observed in the DNS at $\widehat{t}=0.73$ and the contours are shown for the middle $x-z$ plane of the computational 
domain. Just to re-emphasis the point discussed in section 2, it is noted that the laminar flame quantities required for the normalisation are obtained from the premixed flame calculation for the mixture having $T_{u}$ and $P_{u}$ at the chosen time in the DNS. The filtering operation does not alter the relatively larger wrinkling of the flame but the smaller wrinkling is smeared when large filter width is used and this is an expected behaviour. The peak reaction rate is observed to decrease with increase in the filter width. This is because the filtered flame thickness increases with $\Delta^{+}$and thus the peak reaction rate must drop to conserve the overall reaction rate. Similar behaviour is observed for another time, $\widehat{t}=0.91$, and thus those contours are not shown here. However, the peak reaction rate increases with time because of the increase in $T_{u}$ and $P_{u}$ (see Fig. 2). The contours on the right column are for the modeled filtered reaction rate obtained using Eq. (3). This flamelet model seems to capture the spatial variations of the filtered reaction rate and also the flame wrinkles observed in the DNS. The trend of drop in the peak reaction rate with $\Delta^{+}$is also observed for the modelled value as for the DNS results and the comparison shown in Fig. 3 is quite good in general. However, if one makes a closer comparison of the modelled rection rate to those extracted from the DNS then some over predictions can be seen, which is discussed further below. The contours shown in this figure suggest that $\Delta^{+} \leq 0.5$ may be required for isochoric (IC engine) combustion modelling using unstrained flamelets. If this condition is applied for the largest $P_{u}$ and $T_{u}$ expected during the combustion process then it is satisfied automatically for earlier times. Further quantitative evaluation of this combustion model is discussed below. 

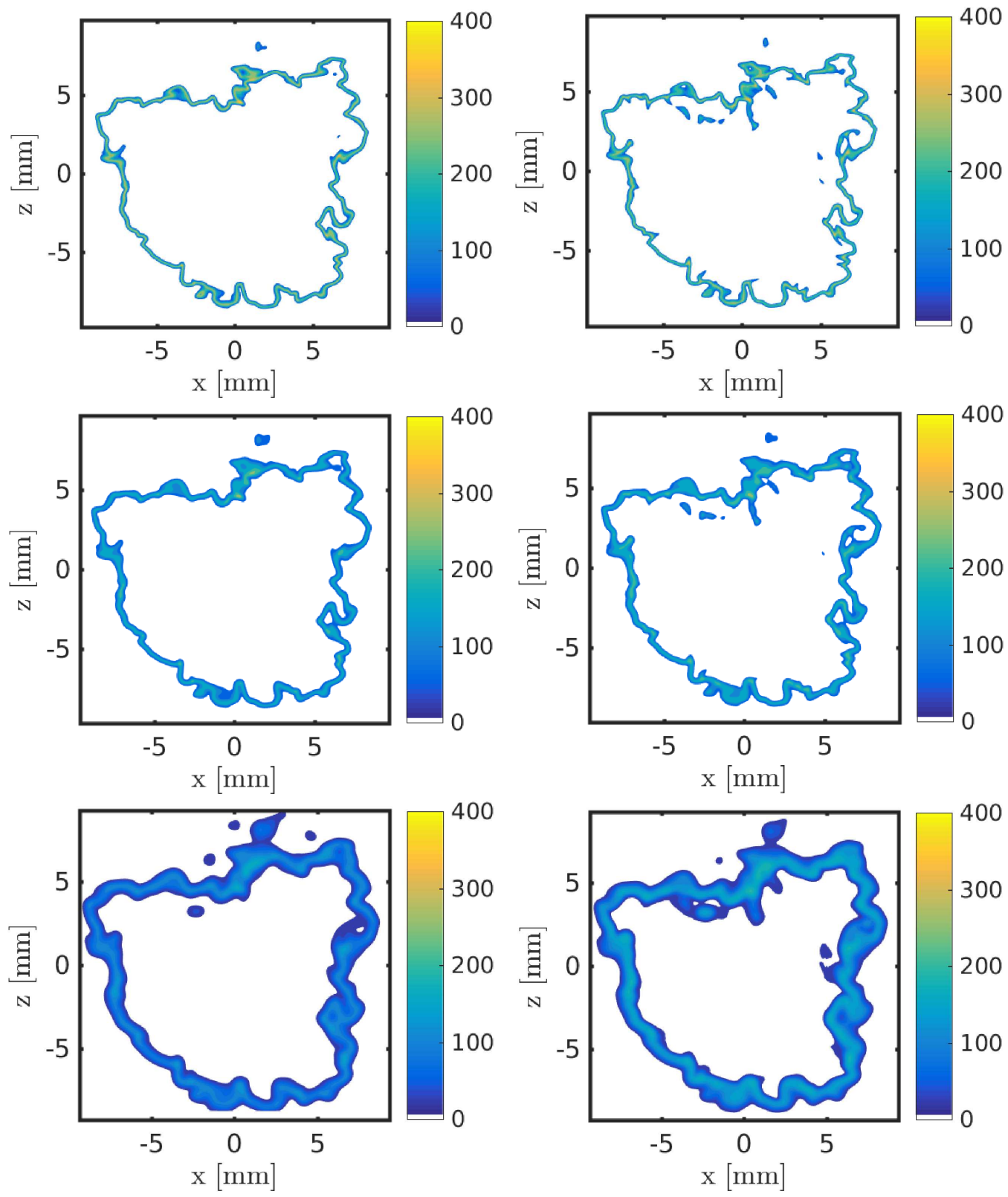

Figure 3: Contours of filtered reaction rate from the DNS (left column) for $\Delta^{+}=0.5,1$ and 2 (from top to bottom) at $\widehat{t}=0.73$ and the corresponding modelled reaction rate (right). The reaction is normalised using $\rho_{u}, s_{L}$ and $\delta_{t h}$. 


\subsection{SGS reaction rate closure}

The reaction rate closure given in Eq. (3) uses unstrained laminar flames to build a look-up table which has $\widetilde{c}$ and $\sigma_{c, s g s}^{2}$ as two controlling variable for a given thermo-chemical condition $\left(T_{u}\right.$ and $\left.P_{u}\right)$. Since the range of these two controlling variables are known the look-up table can be constructed $a$ priori. The top row of Fig. 4 shows the variations of this modelled reaction rate for two instances, $\widehat{t}=0.73$ and 0.91 along with the corresponding filtered reaction rate extracted from the DNS data in the bottom row. The reaction rates are normalised using $\rho_{u}=0.505 \mathrm{~kg} / \mathrm{m}^{3}, s_{L}=11.71 \mathrm{~m} / \mathrm{s}$ and $\delta_{t h}=$ $3.49 \times 10^{-4} \mathrm{~m}$ for $\widehat{t}=0.73$, and $\rho_{u}=0.713 \mathrm{~kg} / \mathrm{m}^{3}, s_{L}=14.36 \mathrm{~m} / \mathrm{s}$ and $\delta_{t h}=2.13 \times 10^{-4} \mathrm{~m}$ for $\widehat{t}=0.91$. Since $T_{u}$ and $P_{u}$ are increasing with time the reactant density and burning velocity increase while the laminar flame thermal thickness decreases. This is the reason why the normalised reaction rates at $\widehat{t}=0.91$ is seen to be smaller than those at an earlier time. Indeed, the dimensional values are larger for the later time.

The modelled normalised reaction rate, $\overline{\dot{\omega}}_{\text {Model }}^{+}$, has its maximum value for $\sigma_{c, s g s}^{2}=0$ which corresponds to quasi-steady and quasi-laminar flames. As the SGS variance increases the reaction rate drops for a given $\widetilde{c}$ value because the burning mode part of the SGS pdf, $\widetilde{p}(0.1 \leq \zeta \leq 0.9)$, decreases. Physically, this implies that the flame fronts are thin and thus the SGS pdf is bimodal. The peak value seen around $\widetilde{c}=0.5$ for a given value of SGS variance is because the reactant mixture is stoichiometric hydrogen-air. A similar behaviour is seen for the DNS results also and these results are constructed by collecting the reaction rate from every point within the flame (identified by using the instantaneous reaction rate to be larger than $5 \%$ of 

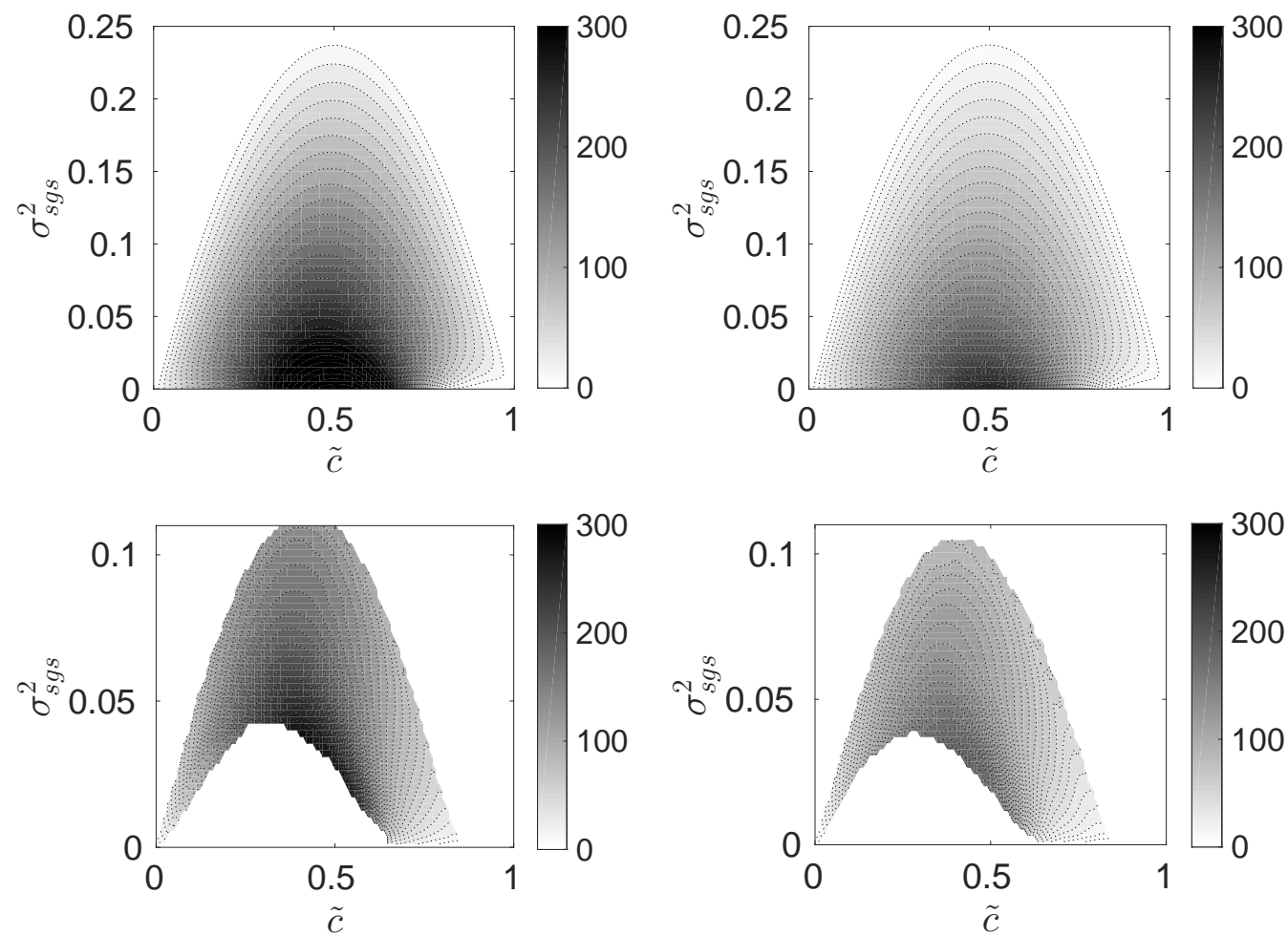

Figure 4: Typical variation of normalised filtered reaction rate from the model (look-up table in the top row) and the DNS values (bottom) for $\Delta^{+}=0.5$. The results are shown for two different times $\widehat{t}=0.73$ (left column) and 0.91 (right).

its maximum value) and then conditionally averaging these samples. The maximum value of $\sigma_{c, s g s}^{2}$ for a given $\widetilde{c}$ is limited by $\widetilde{c}(1-\widetilde{c})$ when the flame front is very thin. The value observed in the DNS is well below 0.25 clearly suggesting that the bimodal limit (thin flame front) is not observed for the conditions of the DNS, despite the initial turbulence characteristics seem to suggest corrugated flamelets regime combustion. This difference is because of the effects of heat release on the local turbulence which has been recognised in many past studies on constant pressure combustion, for example see [3, 47]. 
The DNS results also show that there is a lower limit for $\sigma_{c, s g s}^{2}$ for a given

value of $\widetilde{c}$. The absence of very low $\sigma_{c, s g s}^{2}$ values implies that there are no (in a statistical sense) quasi-steady and quasi-laminar flames in the DNS. A comparison of the results in Fig. 4 shows that the general variation of the filtered reaction rate with $\widetilde{c}$ and $\sigma_{c, s g s}^{2}$ is captured quite well by the unstrained flamelet model.
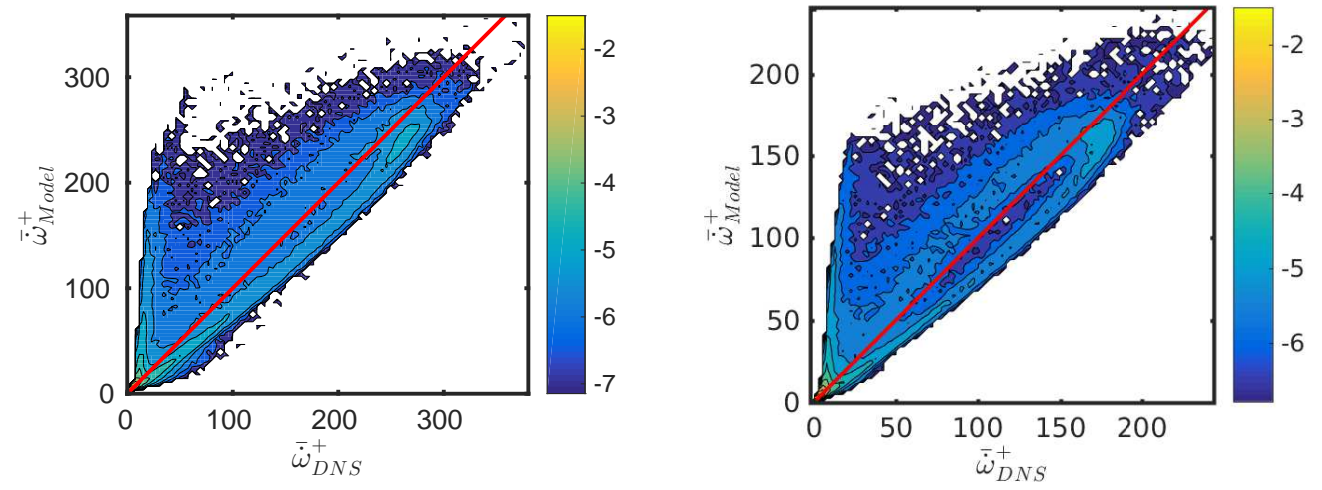

Figure 5: Joint pdf, $\mathcal{P}$, of normalised DNS and modelled filtered reaction rate for $\widehat{t}=$ 0.73 (left) and 0.91 (right). The results are shown for $\Delta^{+}=0.5$. The colour scale is for $\log (\mathcal{P})$.

Figure 5 shows the contours of joint pdf, $\mathcal{P}$, of modelled and DNS values of reaction rates normalised using $\rho_{u}, s_{L}$ and $\delta_{t h}$. The contours are shown for $\Delta^{+}=0.5$. If there is a perfect match between the modelled and DNS values of the filtered reaction rate at every point inside the flame then the all the data points, thus $\mathcal{P}$, would lie along the diagonal line. The joint pdf 330 is shown here rather than the scatter plot of $\overline{\dot{\omega}}_{\text {Model }}^{+}$vs $\overline{\dot{\omega}}_{D N S}^{+}$so that the 
model performance can be evaluated in a statistical sense. The joint pdf contours are observed to align along the diagonal line and the peak of the pdf is close to the origin. It is also worth to note that $\log (\mathcal{P})$ is shown and thus there is four orders of magnitude difference between the brighter and

darker regions. The values of $\log (\mathcal{P})$ having large values of $\overline{\dot{\omega}}_{\text {Model }}^{+}$are smaller than -7 while the peak value is seen to be larger than -2 . Thus, there is a long tail for the larger value of $\bar{\omega}_{\text {Model }}^{+}$, which is not statistically important. Hence, one can conclude that the unstrained flamelet model works quite well for the stoichiometric hydrogen-air premixed combustion at constant volume and the results discussed above offer good support for this. However, the SGS variance of the reaction progress variable needs to be obtained from its transport equation, Eq. (4), in the LES for the reaction rate modelling and thus, one must use closures for $\overline{c \dot{\omega}_{c}}$ and the sub-grid scale SDR, $\widetilde{\epsilon_{c}}$. The former term can be modelled in a manner similar to the unstrained flamelet closure in Eq. (3) and the later quantity can be modelled using Eq. (5), which is evaluated in the discussion below.

\subsection{Evaluation of $\widetilde{\epsilon_{c}}$ closure}

Figure 6 compares the sub-grid scale SDR computed directly from the DNS data and its modelled value obtained using Eq. (5) for two different times. The values are normalised using the respective $s_{L}$ and $\delta_{t h}$. The model parameter $\beta_{c}$ is chosen specifically to get a good correspondence with the DNS data and its value changes with time, 0.6 for $\widehat{t}=0.73$ and 0.3 for 0.91 . This is because, the objective here is to evaluare this model expression by feeding the required quantities, except $\beta_{c}$, obtained directly from the DNS. If the model performs well then all the data points must lie along the diagonal 
line. The results in the figures suggests that the model captures the DNS values quite well for both times and there is some scatter which is typical of turbulent flows involving some inherent randomness. Most of the data points are close to the diagonal line, which is confirmed by constructing the joint pdf of modelled and DNS values of $\widetilde{\epsilon}_{c}^{+}$. This joint pdf is not shown here for brevity. One needs to specify $\beta_{c}$ to use this $\widetilde{\epsilon}_{c}$ model in LES and it can be evaluated using the dynamic procedures given by Eqs. (9) or (10). Before discussing these results, let us understand why $\beta_{c}$ value varies with time.
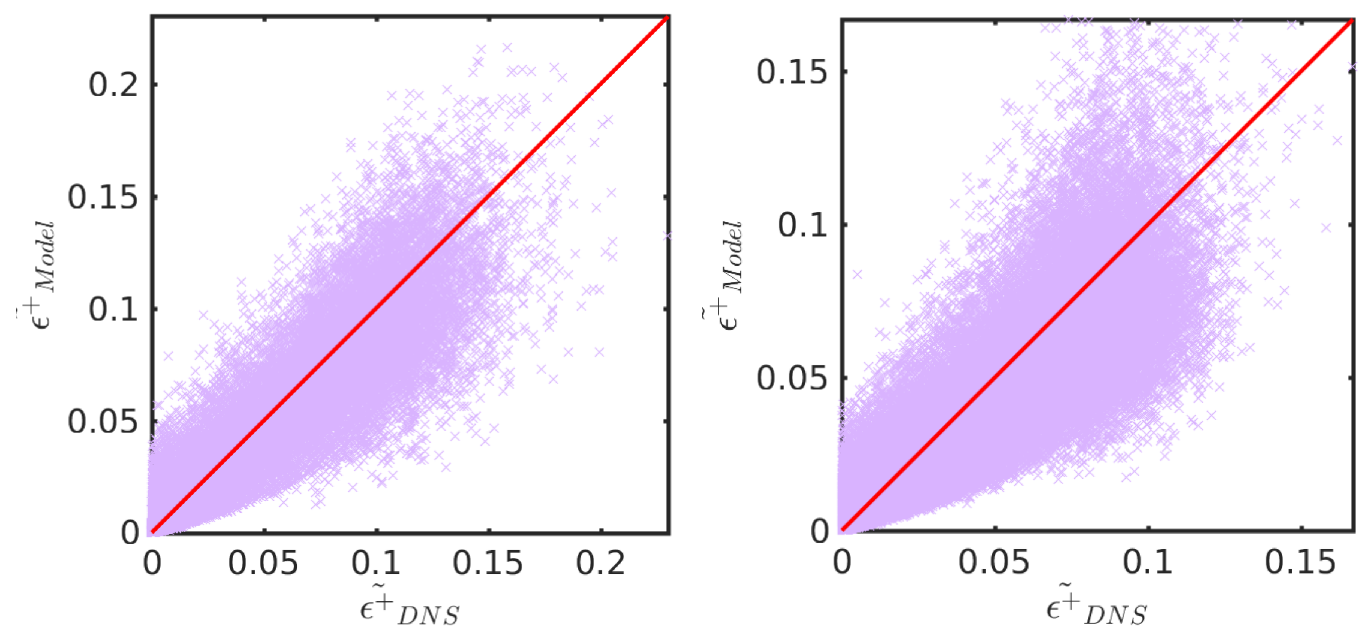

Figure 6: Comparison of DNS and modelled SGS scalar dissipation rate at $\widetilde{t}=0.73$ (left) and 0.91 (right) for $\Delta^{+}=0.5$. The modelled value is obtained using Eq. (5) with $\beta_{c}=0.6$ and 0.3 respectively for $\widetilde{t}=0.73$ and 0.91 .

The parameter $\beta_{c}$ in Eq. (5) is related to the behaviour of $\left(T_{4}-D_{2}\right)$ [48, 49] which involve $\nabla \dot{\omega}, \nabla c$ and $\nabla^{2} c$. The symbols $T_{4}$ and $D_{2}$ are defined as

$$
T_{4}=2 \mathcal{D}\left(\nabla c \cdot \nabla \dot{\omega}_{c}\right) \quad \text { and } \quad D_{2}=2 \rho \mathcal{D}^{2}(\nabla \nabla c: \nabla \nabla c) .
$$


Thus, one sees that $\beta_{c}$ behaviour is strongly related to $\nabla \dot{\omega}, \nabla c$ and $\nabla^{2} c$, which are scale dependant quantities and also the changes in $T$ and $P$ with time in isochoric combustion influence these gradients strongly. There is about 136 percent increase in the peak value of $\dot{\omega}_{c}$ at $\widehat{t}=0.91$ compared to that at $\widehat{t}=0.73$. This is because the temperature and pressure inside the domain increases as the the flame propagates. It is well known that increasing $T$ and $P$ result in thinner flame and higher reaction rate as noted earlier in section 4.1. This consequently results in increase of $\nabla \dot{\omega}, \nabla c$ and $\nabla^{2} c$. Thus, one expects that $\beta_{c}$ will change with time and this change is in addition to the spatial variation at a given time and these changes can only be captured using dynamic procedures in LES. These procedures have been developed in past studies and are briefly reviewed in section 2, Two dynamic methods for evaluating this model parameter are given by Eqs. (9) and (10). The various, including the test filtered, quantities involved in those two equations are obtained directly from the DNS data. The PDFs of this model parameter obtained using the dynamic approaches are shown in Fig. 7 for both the equations. These results are shown for two different times using the samples collected from locations having $\overline{\dot{\omega}}_{c} \geq 0.05\left\langle\dot{\omega}_{c}\right\rangle_{\max }$, where the angle brackets denote the time averaging. This condition allows to pick regions with considerable reaction since the dynamic procedures in Eqs. (9) and (10) do not make sense outside the flame.

385 When the model parameter is assumed to be constant for the test filtering then the range of $\beta_{c}^{*}$ obtained using Eq. (10) is considerably shorter than for $\beta_{c}$ from Eq. (9) as shown in Fig. 7 for $\widehat{t}=0.73$. The most probable values of $\beta_{c}^{*}$ and $\beta_{c}$ are respectively 0.63 and 0.88 . The constant value used for 

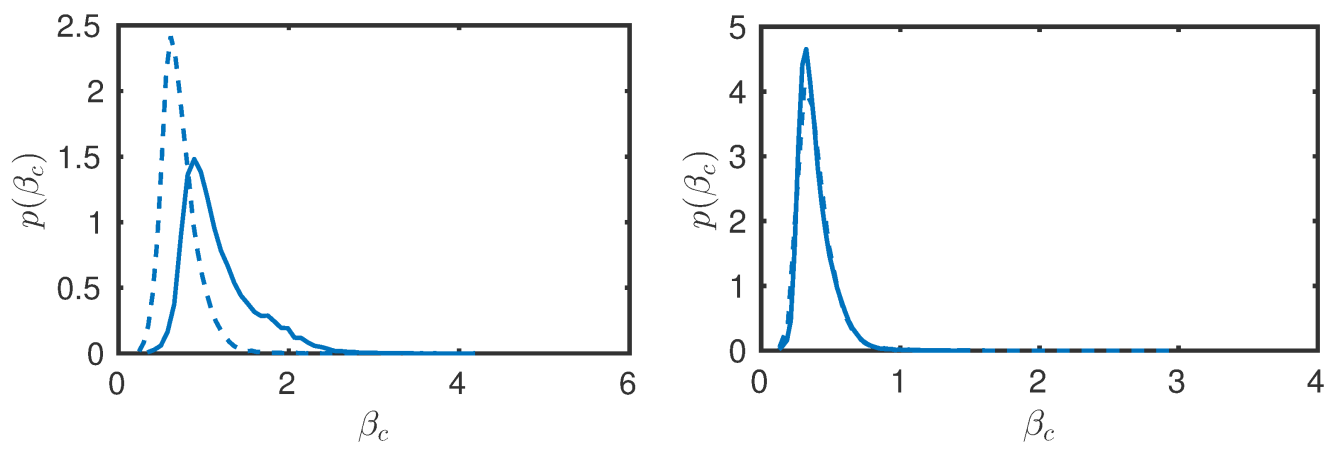

Figure 7: Pdf of $\beta_{c}$ (solid line) and $\beta_{c}^{*}$ (dashed) evaluated using Eqs. (9) and (10) for a filter width of $\Delta^{+}=0.5$. The left frame is for $\widehat{t}=0.73$ and the right is for 0.91 .

the results in Fig. 6 for this time is very close to the most probable value of $\beta_{c}^{*}$. When the reaction is stronger (flame is thinner) then the difference between $\beta_{c}$ and $\beta_{c}^{*}$ is negligible as seen in Fig. 7 for $\widehat{t}=0.91$ and the most probable value is about 0.31 which is almost the same as that used for the corresponding time in Fig. 6. Since the reaction rates are larger at this time the SDR will be larger and thus one gets smaller $\beta_{c}$ values compared to those for $\widehat{t}=0.73$. Thus, it is quite clear that if this model parameter, irrespective of $\beta_{c}$ or $\beta_{c}^{*}$, is chosen carefully then the $\widetilde{\epsilon}_{c}$ model in Eq. (5) works well.

The performance of this model with its parameter obtained using Eqs. (9) and (10) are evaluated in a statistical sense by calculating the joint pdf of the modelled and DNS values of the sub-grid scale SDR. This joint pdf is shown in Fig. 8 for both $\beta_{c}$ and $\beta_{c}^{*}$ evaluations at two different times. The model with $\beta_{c}^{*}$ works well and for the earlier time whereas the SDR model with either $\beta_{c}^{*}$ or $\beta_{c}$ works equally well for later time. There is some over prediction of the SDR however the pdf values for these over predictions are small and thus it can be neglected. It is clear that the SDR model in Eq. (5) with the dynamic procedure in Eq. (10) works well and can be used in LES 

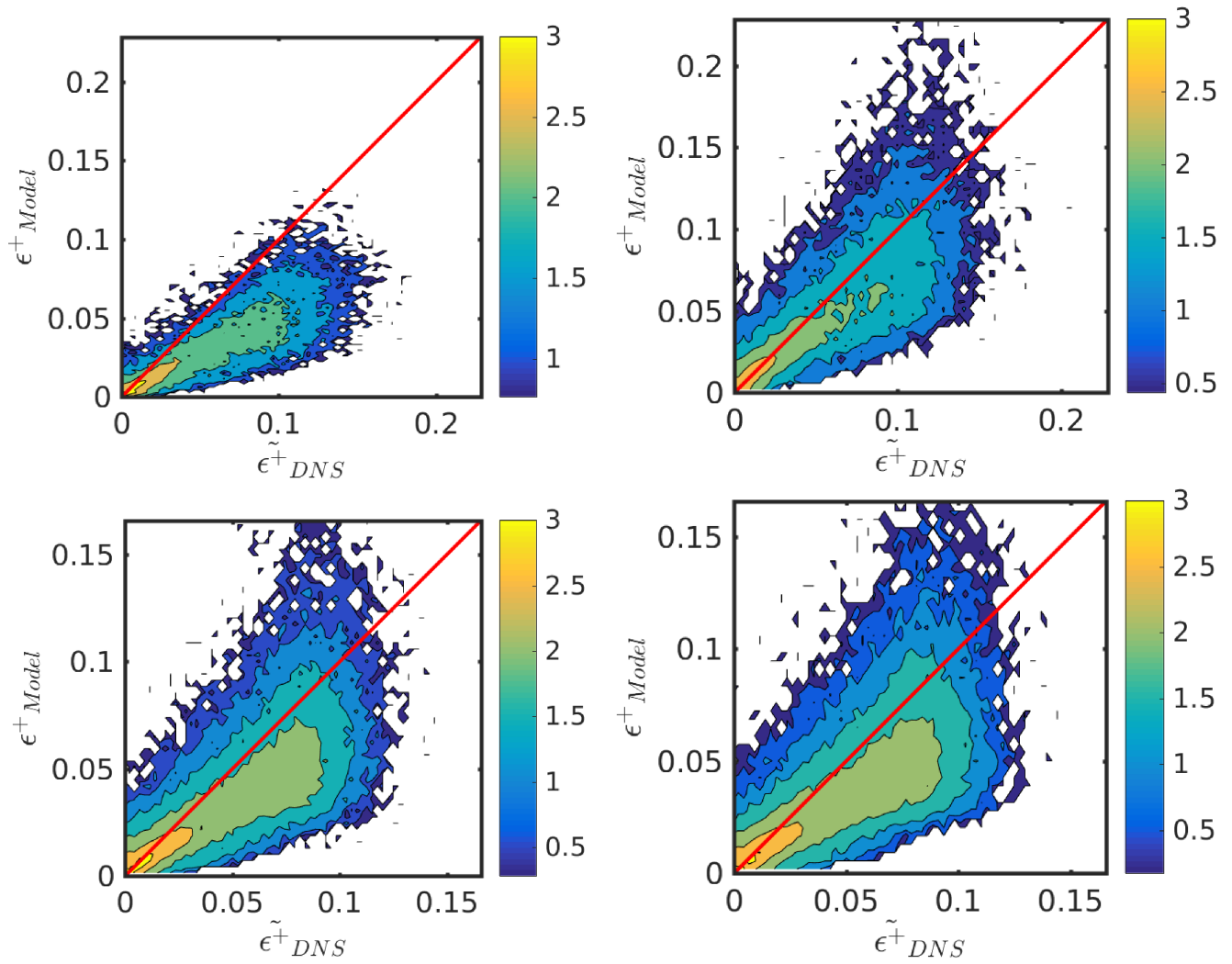

Figure 8: Joint pdf of modelled and DNS values of SDR, normalised using $s_{L}$ and $\delta_{t h}$ appropriately. The results are shown for $\widehat{t}=0.73$ (top row) and 0.91 (bottom) for $\beta_{c}$ obtained using Eq. (9) (left column) and $\beta_{c}^{*}$ from Eq. (10) (right).

of engine simulations, which will be explored in a future study.

\section{Summary and Conclusion}

DNS data of stoichiometric hydrogen-air turbulent premixed flame propagating inside a constant volume chamber has been analysed to shed physical insight on the effects of pressure and temperature rising conditions on flamelets based combustion closure for sub-grid scale reaction rate. The computational domain for the DNS is representative of the combustion chamber 
in an IC engine when its piston is at the top dead centre. The reaction rate closure using FlaRe (Flamelets Revised for physical consistencies) approach is investigated. This approach is based on the unstrained flamelets and gives appropriate emphasis on the SGS variance of reaction progress variable, various physical processes influencing the evolution of this variance and their modelling. Two specific terms, the reaction rate and the SGS scalar dissipation rate, are investigated in detail using the DNS data. Since the temperature and pressure increases with time in constant volume combustion process, the reactions become stronger with time leading to larger dissipation rates and thus the scalar dissipation rate model parameter must be evaluated dynamically. Two variants of a dynamic procedure studied in the past are investigated. It has been shown that the unstrained flamelet closure for the reaction rate and the SDR model in Eq. (5), along with the dynamic procedure in Eq. (10), work quite well for constant volume combustion of stoichiometric hydrogen-air mixture. Although this modelling approach using URANS paradigm has been explored in the past [33, 34] its application in LES framework for engine flows will be explored in future.

\section{Acknowledgements}

The support of KACST is acknowledged by G.G. and N.S. The funding from Qualcomm European Research Studentship in Technology is acknowledged by N.A.K.D. 
[1] Tanahashi, M., Fujimura, M., Miyauchi, T. Coherent fine scale eddies in turbulent premixed flames. Proc. Combust. Inst. 2000;28:529-535.

[2] Shimura, M., Fukushima, N., Nada, Y., Tanahashi, M., Miyauchi, T. Radical fingering in turbulent premixed flame classified into thin reaction zones. Proc. Combust. Inst. 2013;34:1383-1391.

[3] Poludnenko, A. Y., Oran, E. S. The interaction of high-speed turbulence with flames: Global properties and internal flame structure. Combust. Flame 2010;157:995-1011.

[4] Shim, Y., Tanaka, S., Tanahashi, M., Miyauchi, T. Local structure and fractal characteristics of H2-air turbulent premixed flame. Proc. Combust. Inst. 2011;33:1455-1462.

[5] Minamoto, Y., Fukushima, N., Tanahashi, M., Miyauchi, T., Dunstan, T. D., Swaminathan, N. Effect of flow-geometry on turbulence-scalar interaction in premixed flames. Phys. Fluids 2011;23:125107.

[6] Gruber, A., Sankaran, R., Hawks, E. R., Chen, J. H. Turbulent flamewall interaction: a direct numerical simulation study. J. Fluid Mech. 2010;658:5-32.

[7] Tanaka, S., Shimura, M., Fukushima, N., Tanahashi, M., Miyauchi, T. DNS of turbulent swirling premixed flame in a micro gas turbine combustor. Proc. Combust. Inst. 2011;33:3293-3300.

[8] Wang, H., Luo, K., Qiu, K., Lu, S., Fan, J. A DNS study of hydro455 gen/air swirling premixed flames with different equivalence ratios. Int. J. Hydrogen Energy 2012;37:5246-5256. 
[9] Minamoto, Y., Aoki, K., Tanahashi, M., Swaminathan, N. DNS of swirling hydrogen-air premixed flames. Int. J. Hydrogen Energy 2015;40:13604-13620.

460

465

[15] Peters, N., Turbulent Combustion, Cambridge University Press, 2000.

[16] Poinsot, T., Veynante, D., Theoretical and Numerical Combustion, 2nd ed., R. T. Edwards, Philadelphia, PA, USA, 2005. 
[17] Swaminathan, N., Bray, K. N. C. Fundamentals and challenges. in: Swaminathan, N., Bray, K. N. C. (Eds.), Turbulent Premixed Flames, Cambridge University Press, Cambridge, UK, 2011, pp. 1-33.

[18] Sarli, V. D., Di, B. A., G., R. Sub-grid scale combustion models for large eddy simulation of unsteady premixed flame propagation around obstacles. J. Hazardous Materials 2010;180:71-78.

[19] Sarli, V. D., Di, B. A., G., R. Large eddy simulation of transient premixed flame-vortex interactions in gas explosions. Chem. Engg. Sci. 2012;71:539-551.

[20] Sarli, V. D., Di, B. A. Sensitivity to the presence of the combustion submodel for large eddy simulation of transient premixed flame-vortex interactions. Ind. Eng. Chem. Res. 2012;51:7704-7712.

[21] Sarli, V. D., Di, B. A. Effects of non-equidiffusion on unsteady propagation of hydrogen-enriched methane/air premixed flames. J. Hydrogen Energy 2013;38:7510-7518.

[22] Haworth, D. C. Large-eddy simulation of in-cylinder flows. Oil Gas Sci. Technol. 1999;54:175-185.

[23] Haworth, D. C. A review of turbulent combustion modeling for multidimensional in-cylinder CFD. SAE Technical Paper 2005-01-0993 $2005 ;: 899-927$.

[24] Drake, M. C., Haworth, D. C. Advanced gasoline engine development using optical diagnostics and numerical modeling. Proc. Combust. Inst. 2007;31:99-124. 
[25] Kolla, H., Swaminathan, N. Strained flamelets for turbulent premixed flames, I: Formulation and planar flame results. Combust. Flame 2010;157:943-954.

505

510

[32] Ahmed, I., Simulation of turbulent flames relevant to spark-ignition engines, Ph.D., University of Cambridge, Cambridge, UK, 2014. 
[33] Ahmed, I., Ghiasi, G., Raj, A. G. S., Swaminathan, N., Koch, J., Steurs, K., Wright, Y. M. Spark ignition engine simulation using a flamelet based combustion model. SAE Technical Paper 2015-24-2402 2015;:113.

[34] Ghiasi, G., Ahmed, I., Wright, Y. M., Koch, J., Swaminathan, N. Sensitivity of flamelet combustion model to flame curvature for IC engine application. SAE Technical Paper 2017-24-0038 2017;:1-10.

[35] Langella, I., Swaminathan, N. Unstrained and strained flamelets for LES of premixed combustion. Combust. Theory Model. 2016;7830:1-31.

[36] Langella, I., Swaminathan, N., Pitz, R. W. Application of unstrained flamelet SGS closure for multi-regime premixed combustion. Combust. Flame 2016;173:161-178.

[37] Chen, Z., Ruan, S., Swaminathan, N. Large eddy simulation of flame edge evolution in a spark-ignited methane-air jet. Proc. Combust. Inst. 2017;36:1645-1652.

[38] Gao, Y., Chakraborty, N., Swaminathan, N. Dynamic closure of scalar dissipation rate for large eddy simulations of turbulent premixed combustion: A direct numerical simulations analysis. Flow, Turbul. Combust. 2015;95:775-802.

[39] Langella, I., Swaminathan, N., Gao, Y., Chakraborty, N. Assessment of dynamic closure for premixed combustion large eddy simulation. Combust. Theory Model. 2015;19:628-656. 
[40] Dunstan, T. D., Minamoto, Y., Chakraborty, N., Swaminathan, N. Scalar dissipation rate modelling for large eddy simulation of turbulent premixed flames. Proc. Combust. Inst. 2013;34:1193-1201.

[41] Kolla, H., Rogerson, J. W., Chakraborty, N., Swaminathan, N. Scalar dissipation rate modeling and its validation. Combust. Sci. Technol. $2009 ; 181: 518-535$.

[42] Gao, Y., Chakraborty, N., Swaminathan, N. Algebraic closure of scalar dissipation rate for large eddy simulations of turbulent premixed combustion. Combust. Sci., Technol. 2014;186:1309-1337.

[43] Langella, I., Swaminathan, N., Gao, Y., Chakraborty, N. LES of premixed combustion: Sensitivity to SGS velocity modelling. Combust. Sci. Technol. 2017;189:43-78.

[44] Langella, I., Large Eddy Simulation of premixed combustion using flamelets, PhD thesis, University of Cambridge, Cambridge, UK, 2015. URL: https://www.repository.cam.ac.uk/handle/1810/254303.

[45] Gutheil, E., Balakrishnan, G., Williams, F. A. Structure and extinction of hydrogen-air diffusion flames. in: Peters, N., Rogg, B. (Eds.), Reduced Kinetics Mechanisms for Applications in Combustion Systems, SpringerVerlag, Berlin,, 1993, pp. 177-195.

[46] Pope, S. B., Turbulent Flows, Cambridge University Press, Cambridge, UK, 2000.

[47] Swaminathan, N., Bray, K. N. C. Effect of dilatation on scalar dissipation in turbulent premixed flames. Combust. Flame 2005;143:549-565. 
[48] Gao, Y., Chakraborty, N., Swaminathan, N. Scalar dissipation rate transport and its modeling for large eddy simulations of turbulent premixed combustion. Combust. Sci. Technol. 2015;187:362-383.

[49] Chakraborty, N., Rogerson, J. W., Swaminathan, N. A priori assessment of closures for scalar dissipation rate transport in turbulent premixed flames using direct numerical simulation. Phys. Fluids 2008;20:45106. 